\title{
The Use of Carbon Quantum Dot as Alternative of Stannous Chloride Application in Radiopharmaceutical Kits
}

\author{
Seyedeh Masoumeh Ghoreishi ${ }^{D},{ }^{1}$ Atena Najdian $\left(\mathbb{D},{ }^{2}\right.$ Sina Yadegari $\left(\mathbb{D},{ }^{2}\right.$ \\ Mohammad Seyedhamzeh (D), ${ }^{2}$ Maziyar Etemadzade $\left(\mathbb{D},{ }^{3}\right.$ Mehdi Mirzaei $\left(\mathbb{D},{ }^{4}\right.$ \\ Shahin Hadadian $\mathbb{D}^{5},{ }^{5}$ Zahra Alikhani ${ }^{(D)}{ }^{6}$ and Mehdi Shafiee Ardestani ${ }^{2}{ }^{2}$ \\ ${ }^{1}$ Cellular and Molecular Biology Research Center, Health Research Institute, Babol University of Medical Sciences, Babol, Iran \\ ${ }^{2}$ Department of Radiopharmacy, Faculty of Pharmacy, Tehran University of Medical Sciences, Tehran, Iran \\ ${ }^{3}$ Department of Biology, Tehran Medical Branch, Islamic Azad University, Tehran, Iran \\ ${ }^{4}$ Iran Ministry of Health and Medical Education, Deputy Ministry for Education, Tehran, Iran \\ ${ }^{5}$ Nanobiotechnology Department, Pasteur Institute of Iran, Tehran, Iran \\ ${ }^{6}$ Department of Microbiology, Kerman Islamic Azad University, Kerman, Iran
}

Correspondence should be addressed to Mohammad Seyedhamzeh; m.s.hamzeh@gmail.com, Maziyar Etemadzade; etemadzademazyar97@gmail.com, and Mehdi Shafiee Ardestani; shafieeardestani@gmail.com

Received 6 June 2020; Revised 10 September 2020; Accepted 15 October 2020; Published 6 November 2020

Academic Editor: Laurent M. Riou

Copyright (C) 2020 Seyedeh Masoumeh Ghoreishi et al. This is an open access article distributed under the Creative Commons Attribution License, which permits unrestricted use, distribution, and reproduction in any medium, provided the original work is properly cited.

\begin{abstract}
Even today, technetium-99m is a radionuclide choice for diagnostic in nuclear medicine. The unique chemical and physical properties of technetium-99m make it suitable as an available radionuclide in many centers. In this study, we examined the potential of CQD as a reducing agent in the MDP kit. Citric acid-derived CQD was synthesized and confirmed by FT-IR, TEM, UV-Vis, XPS, and surface analysis. No cytotoxicity was observed by the MTT assay. They were reducing properties of the CQD confirmed by fluorescence microscopy. The MDP kit is prepared by evaluating different parameters that affect the radiolabeling yield, including ligand, time, and CQD. The optimum amount of each parameter is obtained by Box-Behnken software. Finally, fluorescent spectroscopy, SPECT imaging, and biodistribution study showed that CQD reduces technetium-99m. Accumulation of radiotracer in the femur showed that CQD could be used in a radiopharmaceutical kit.
\end{abstract}

\section{Introduction}

Nowadays, nuclear medicine has attracted much attention due to a multidisciplinary specialty that uses a radioactive tracer to study physiological processes and treat diseases noninvasively [1]. In contrast to anatomical imaging agents (CT, MRI, and ultrasound), which are dependent on the size of abnormality [2], nuclear medicine methods (SPECT and PET) show quantitative functional information [3]. Thus, abnormality can be detected at their initial stage. Since early detection of diseases is essential to the physician to choose a better medical treatment, nuclear medicine plays a critical role in medical specialties $[1,4]$.

Although positron emitter radionuclide has been considered lately, over $70 \%$ of radiotracers are still labeled with single isotope. Among SPECT radioisotopes, technetium$99 \mathrm{~m}$ has been used mostly in the nuclear medicine diagnostic $[5,6]$. Gamma-ray with suitable energy, availability of $99 \mathrm{Mo} / 99 \mathrm{mTc}$ generator, appropriate physical half-life $(6 \mathrm{~h})$, and fast complex formation are the main reasons for the continuous use of technetium-99m. Gamma-ray $(140 \mathrm{keV})$, ideal for SPECT camera, can be injected in low doses [7]; 6-hour half-life provides the appropriate time for the preparation of radiopharmaceuticals and administration to and biodistribution in the patient body, and also it is suitable to accumulate in the targeted organs. New technetium-99m radiopharmaceuticals have been developed, while technetium chemistry has been advanced. The abovementioned leads us to believe that 
radiopharmaceuticals labeled with technetium-99m will continue to play an essential role in nuclear medicine [8].

In most labeling process, technetium- $99 \mathrm{~m}$ must be reduced from the +7 valence state. Stannous chloride is a joint reducing agent that is used routinely in radiopharmaceutical kits. Since technetium-99m is usually used for labeling pharmaceuticals, research on the reducing agent for reduction of the technetium- $99 \mathrm{~m}$ is more attractive [9]. Although stannous chloride is a potent reducing agent, it is oxidized easily by oxygen in the atmosphere or solution. Thus, the efficiency of the labeling process decreases. Also, for kit preparation, $\mathrm{SnCl}_{2}$ is dissolved in acidic media, commonly hydrochloric acid. This feature limits its application when the alkaline environment is needed for complex formation. Therefore, research on the new reducing agent can be more practical [5].

Nanoparticles have attracted much attention due to their significant characteristics. So they have been used in many fields, including biosensors, medical and pharmaceutical science, catalysis, and engineering [10-12]. Its applications in medical and pharmaceutical science show significant perspective [13]. For example, nanoparticles are utilized as a carrier for drug delivery [14-16]. In this regard, a large surface area compared to their volume, which is a potential feature of nanoparticles, causes a reduction in the dose of nanoparticles [17, 18]. Among these, carbon quantum nanodots (CQDs) have been introduced as reducing agents for the synthesis of metal nanoparticles. Gholinejad and coworkers reported that magnetic $\mathrm{Fe} 3 \mathrm{O} 4$ modified with CQDs was used for the reduction of PdII and the formation of PdNPs [19]. In 2017, Amjadi and coworkers stated that glucose-derived CQDs had both reducing and stabilizing properties. They synthesized new CQD/Ag nanocomposites as a colorimetric agent to detect trace amounts of methimazole in urine [20]. In this framework, Liu and coworkers prepared CQDs derived from polyethyleneimine (PEI) as reducing and stabilizing agent. The synthesized CQDs presented surprising reducibility [21]. Song et al. developed CQDs prepared with chitosan as both a reducing and stabilizing agent which was further used to synthesize AuNPs for the detection of iodine ions [22].

Since studies on all aspects of ${ }^{99 \mathrm{~m}} \mathrm{Tc}$-radiopharmaceuticals have been impressive all time, in this work, we researched a reducing agent that has a vital role in the technetium-99m labeling process. CQD was used as a reducing agent in the MDP kit. The study aimed to determine whether CQD has a reducing effect on technetium-99m or not. The obtained data supported our hypothesis that CQD can act as a reducing agent in the radiopharmaceutical kit.

\section{Material and Method}

Citric acid, ethylenediamine, and urea were purchased from Merck. Methylene phosphonic acid and MTT powder were purchased from Sigma-Aldrich. The typical cell line HEK293 was obtained from the Pasteur Institute (Tehran, Iran). All experiments were based on the animal ethics committee. Three mice were used for each experiment. An infrared spectrum was measured using the Perkin Elmer Spectrum
BX-II spectrometer. Fluorescence and UV-Vis spectra were measured by a Varian spectrometer. SEM and TEM were performed by using TESCAN and ZEISS instruments. XPS was done to study elemental composition and structural purity of CQDs by JEOL. All studies were repeated three times. Studies were directed in line with the principles of the Declaration of Tehran University of Medical Sciences with the number: IR.TUMS.TIPS.REC.1398.021. Statistical analysis was performed by Box-Behnken and MATLAB software, and $P<0.05$ was considered statistically significant. Briefly, synthesis of the CQD was performed as mentioned below: (1) CQD was synthesized by a mixture of citric acid $(0.524 \mathrm{~g})$ and urea $(0.25 \mathrm{~g})$ in distilled water $(10 \mathrm{~mL})$. The mixture was stirred for $30 \mathrm{~min}$ at room temperature. After that, the product was transferred into the autoclave at 180 centigrade degrees for 6 hours. Finally, larger particles (particles have a size of more than $10 \mathrm{~nm}$ ) were separated by centrifugation (11200 $\mathrm{g}$ for $15 \mathrm{~min}$ ). The obtained CQD was analyzed by FT-IR, fluorescence, and UV-Vis spectroscopy. Also, TEM and XPS were done to evaluate the synthesized CQD. (2) For determination, the safety of the final product cytotoxicity (MTT assay) was examined. The MTT cell proliferation assay measures the cell proliferation rate and, conversely, when metabolic events lead to apoptosis or necrosis, the reduction in cell viability was observed. The MTT assay was done on the HEK-293 cell line according to the published article $[23,24]$. Media from cell cultures were discarded. Then, $50 \mu \mathrm{L}$ of serum-free media and $50 \mu \mathrm{L}$ of MTT solution were added into each well and the plate was incubated at $37^{\circ} \mathrm{C}$ for 3 hours. After incubation, $150 \mu \mathrm{L}$ of MTT solvent was added into each well. Finally, the absorbance was read at $\mathrm{OD}=590 \mathrm{~nm}$. (3) The radiopharmaceutical kit was produced for more examination. For this purpose, MDP (10 mg), ascorbic acid $(1.8 \mathrm{mg})$, and CQD $(100 \mu \mathrm{L}$ of $2 \mathrm{mg} / 1 \mathrm{ml})$ were dissolved in distilled water and then lyophilized. Radiolabeling was done by adding a technetium-99m (370 MBq) and shaken for $15 \mathrm{~min}$. (4) Quality control was performed to evaluate the radiochemical purities (RCPs) in the labeled kit. Impurities were determined by thin-layer chromatography (TLC). Methyl ethyl ketone and sodium acetate were chosen as a mobile phase. (5) For further investigation, in vivo study was done. The anesthetized mice were injected through the tail vein, and then SPECT/CT imaging was done. Finally, the biodistribution of radiotracer was evaluated by sacrificing injected mice and counting each organ.

\section{Result and Discussion}

3.1. Analysis. FT-IR was used to characterize the surface functional groups. The result is shown in Figure 1. Peaks that appeared at $3200-3500 \mathrm{~cm}^{-1}$ belong to the hydroxyl group. $\mathrm{H}-\mathrm{N}$ stretching band presented at about $3000-3300 \mathrm{~cm}^{-1}$. Peaks that appeared at 1400 and $1600 \mathrm{~cm}^{-1}$ belong to $\mathrm{H}-\mathrm{C}$ and carbonyl group, respectively. Bands at $1120 \mathrm{~cm}^{-1}$ and $1342 \mathrm{~cm}^{-1}$ can be assigned as C-N and C-O groups [25]. The emission and excitation properties of the obtained carbon dot were studied by fluorescence spectroscopy at a range of 200-800 nm wavelengths (Figure 2). Carbon quantum dots 


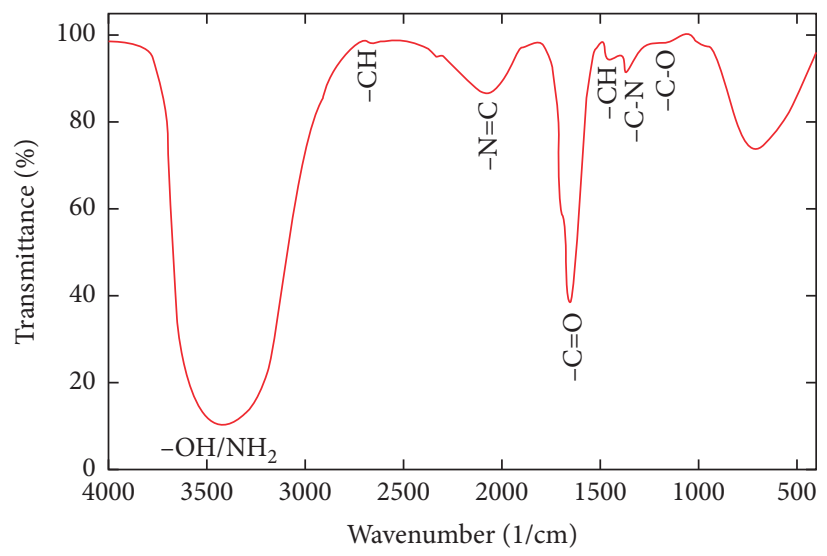

FIGURE 1: FT-IR of CQD.

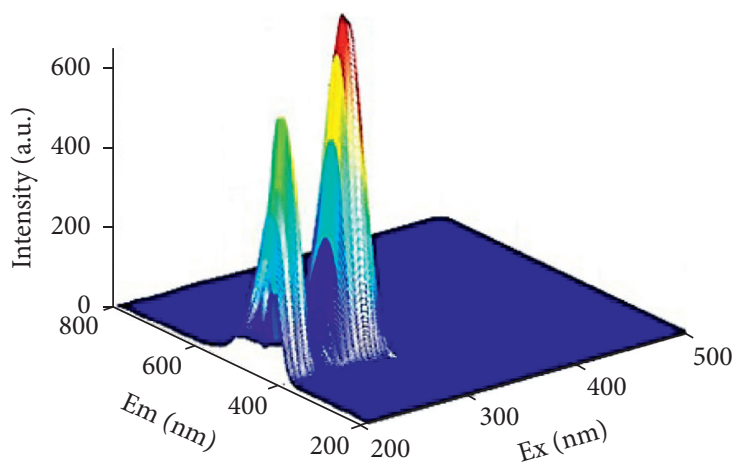

(a)

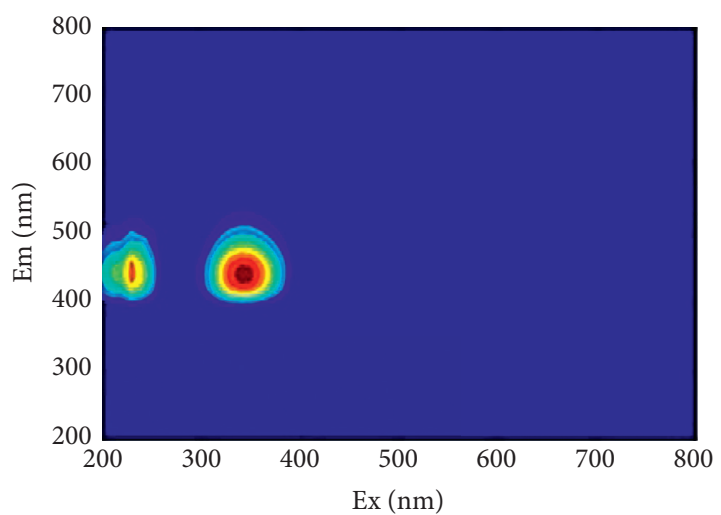

(b)

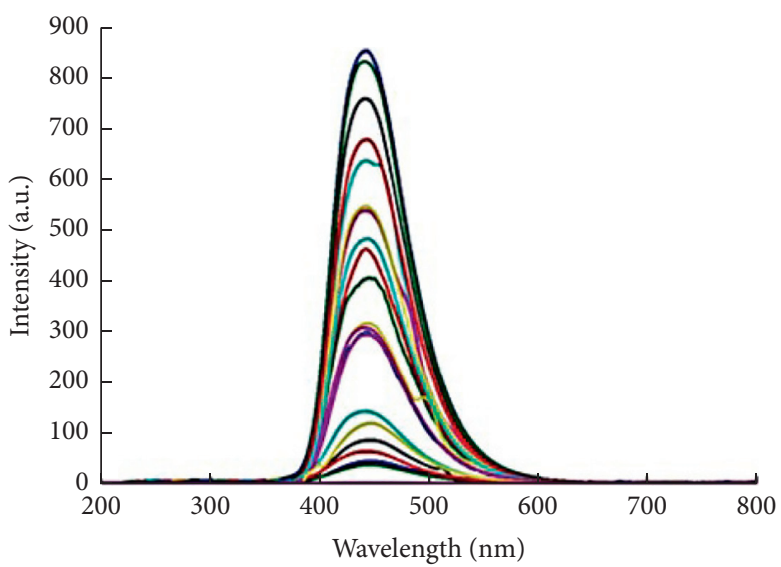

(c)

Figure 2: Fluorescence spectroscopy of CQD.

were excited and emitted in the range of $200-400 \mathrm{~nm}$ and 400-550 nm, respectively (Figures 2(a) and 2(b)). Also, during the excitation at 245 and $382 \mathrm{~nm}$, they showed the maximum emission, which is marked in two separate peaks. The emission of these particles is also independent of excitation changes (Figure 2(c)). It is evident that the maximum intensity of emission is $442 \mathrm{~nm}$ related to $380 \mathrm{~nm}$ excitation wavelength. The CQD CIE chromaticity diagram shows that this compound shows blue color (Figure 3). UVVis absorption spectrum of CQD is presented in Figure 4. CQD showed a peak at $260 \mathrm{~nm}$ and at about $380 \mathrm{~nm}$ related to $\pi-\pi^{*}$ and $\pi-n^{*}$ transitions, which are defined as $\mathrm{C}=\mathrm{C}$ and $\mathrm{C}=\mathrm{O}$ transportation. The size and morphology of CQD were determined by TEM (Figure 5). The result showed that CQD had a spherical shape in $1.2-2.7 \mathrm{~nm}$ size range with an average diameter of $1.87 \pm 0.331 \mathrm{~nm}$. Elemental mapping 


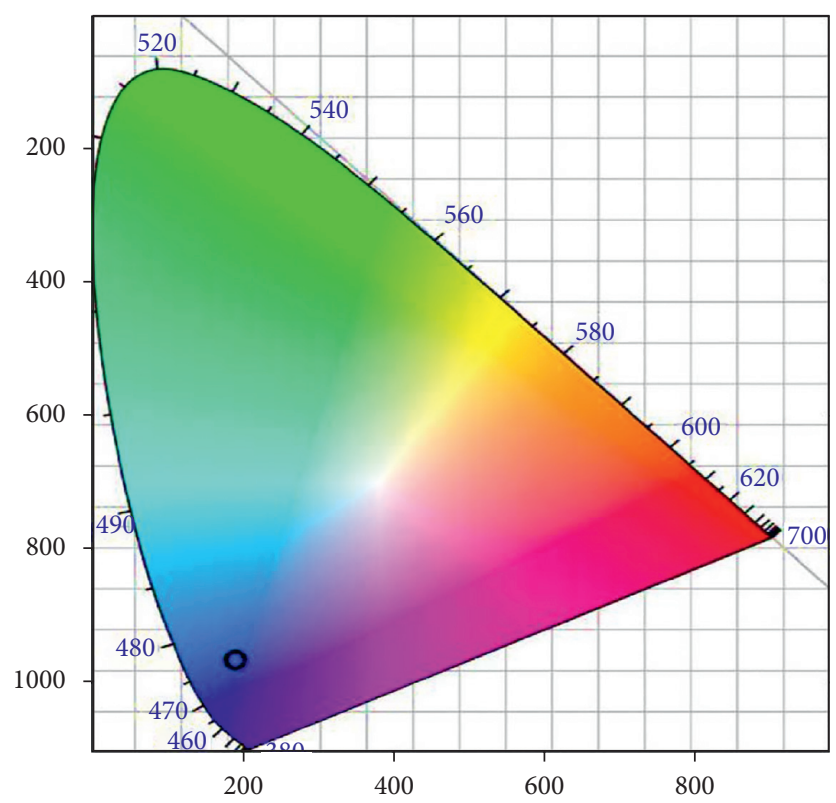

Figure 3: Chromaticity of CQD.

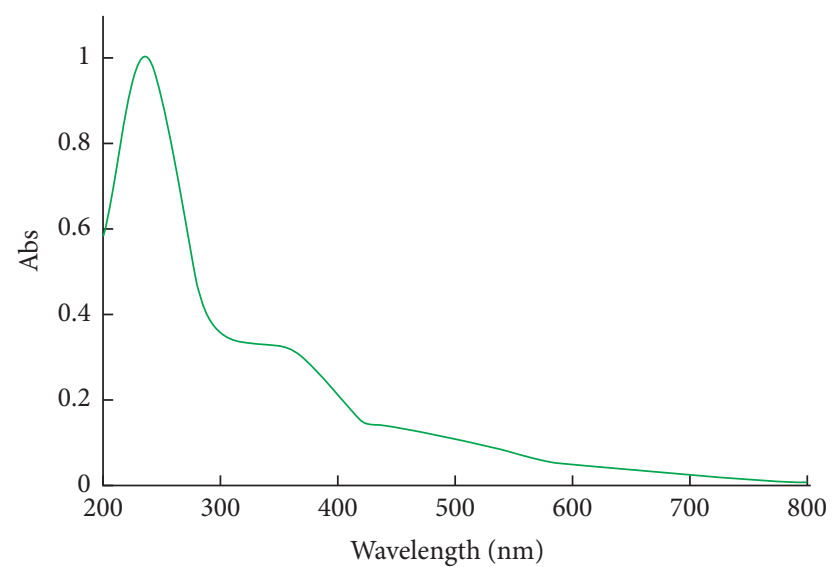

Figure 4: UV-Vis spectroscopy of CQD.

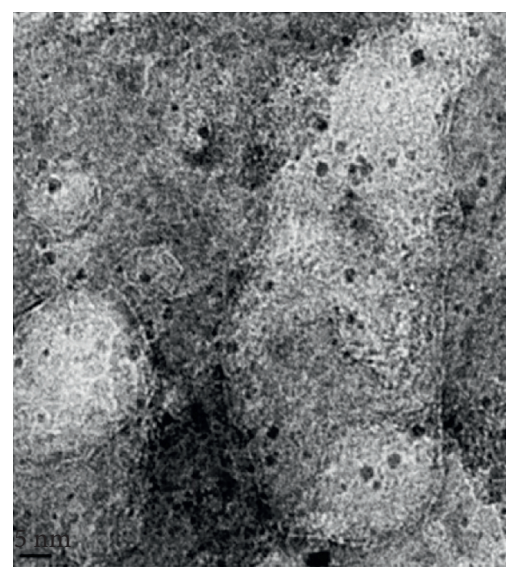

(a)

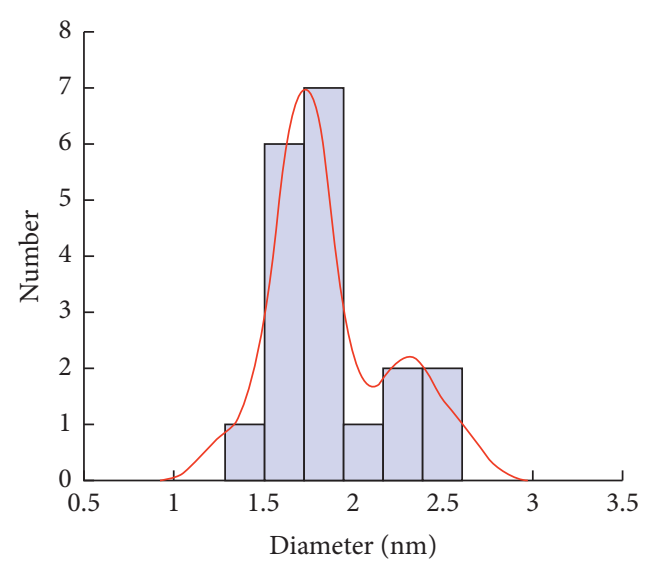

(b)

Figure 5: TEM of CQD. 

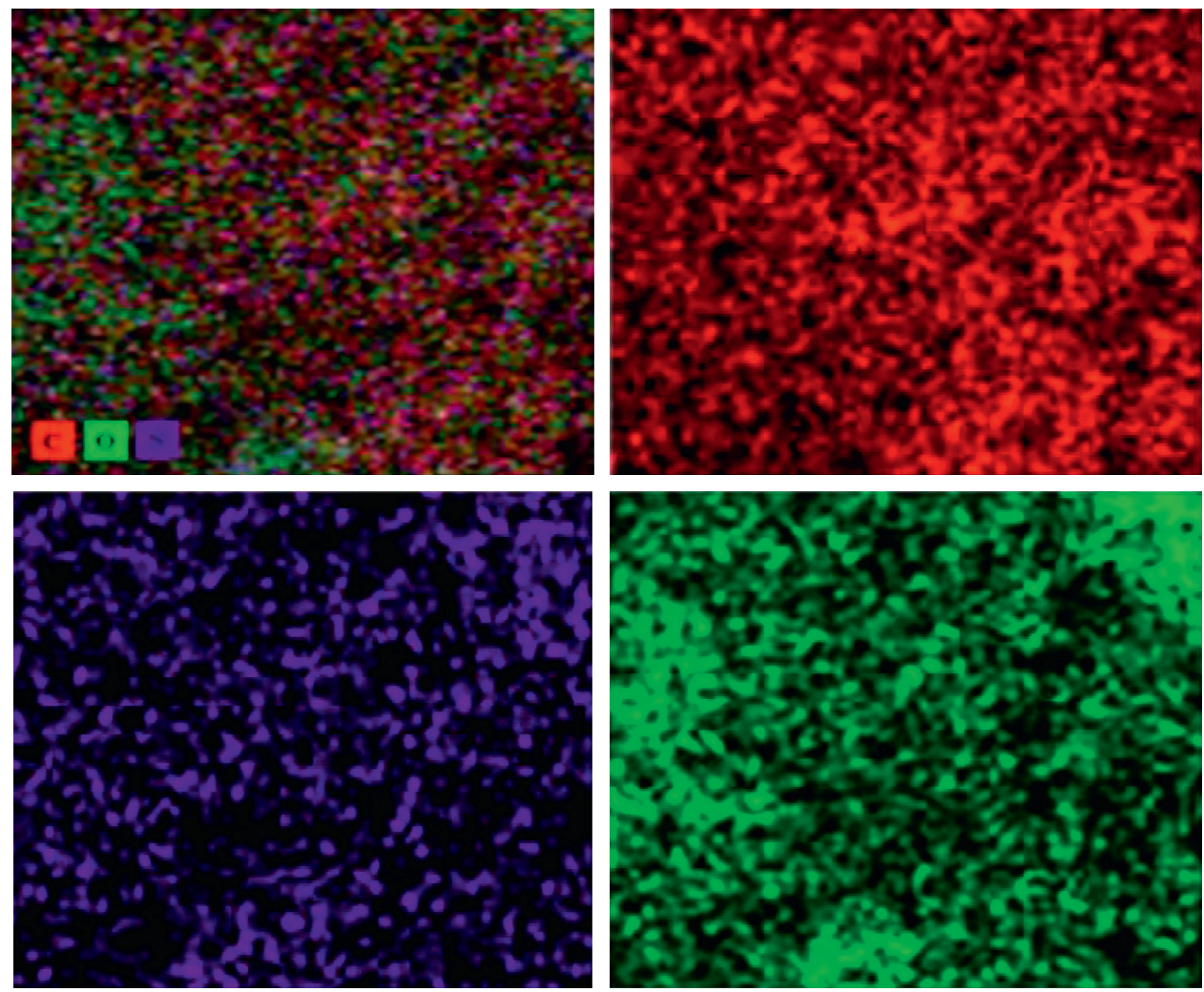

FIgURE 6: Surface analysis of CQD.

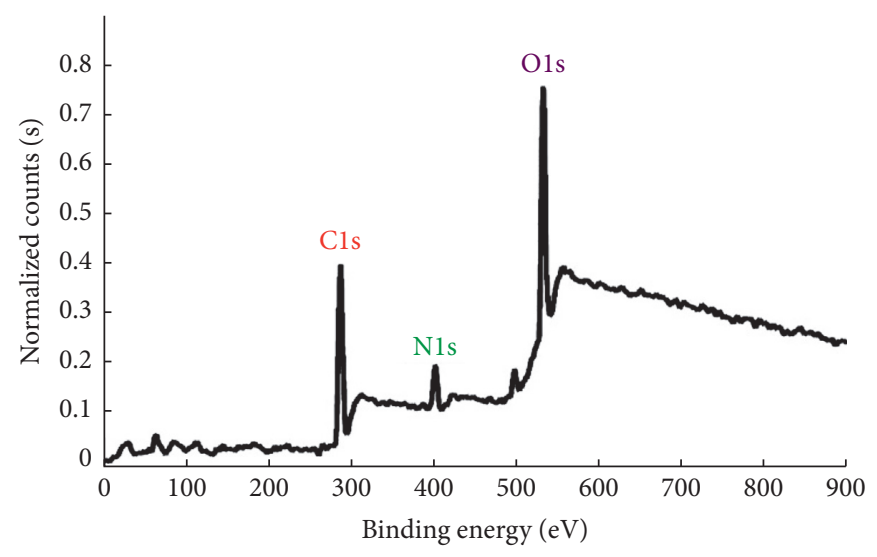

(a)

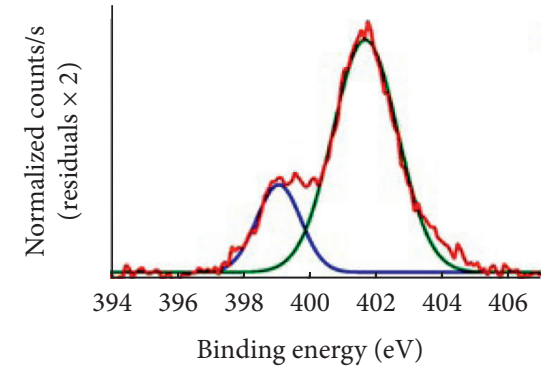

(c)

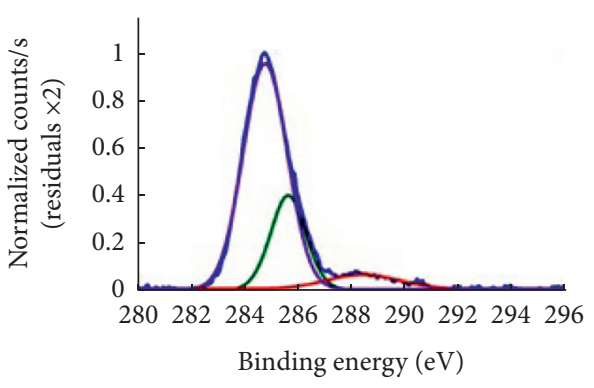

(b)

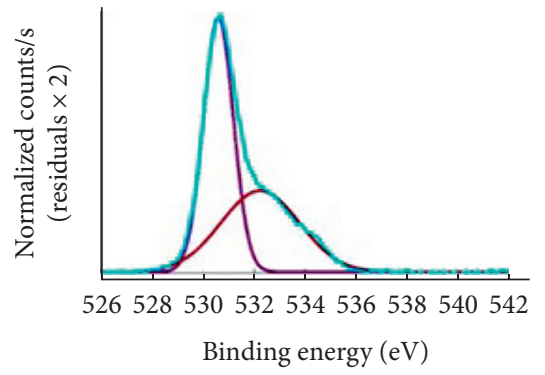

(d)

FIGURE 7: XPS analysis of CQD. 


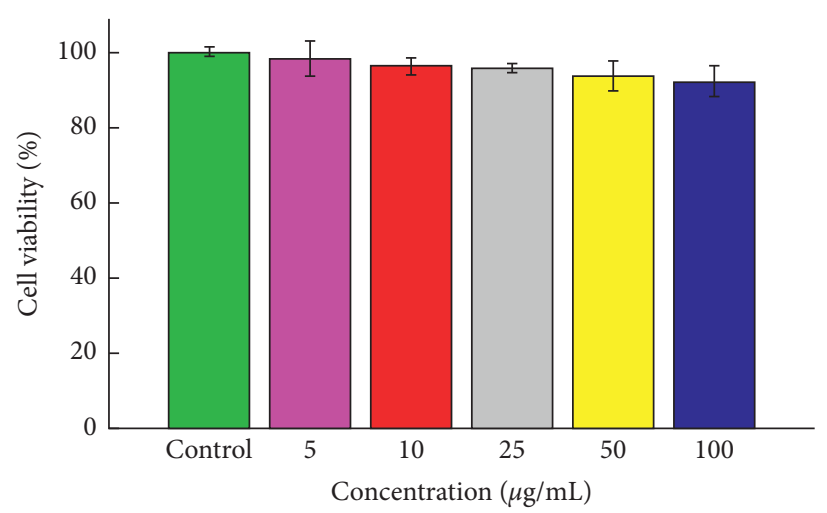

Figure 8: Cytotoxicity assay of CQD on HEK-293 cell line ( $P$ value $<0.05)$.

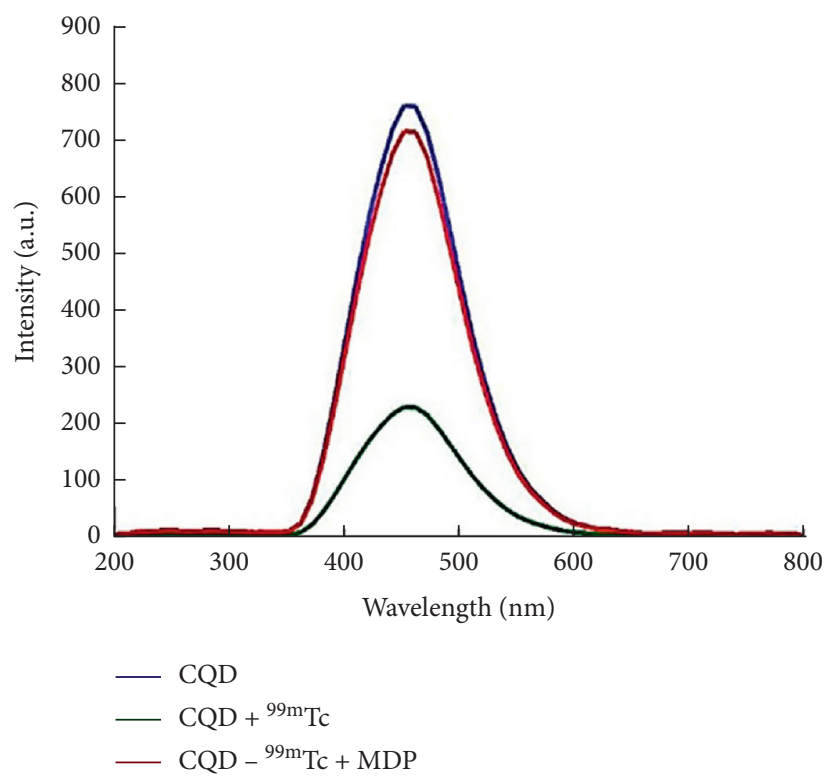

FIGURE 9: Reducing potential of CQD determined by fluorescence spectroscopy.

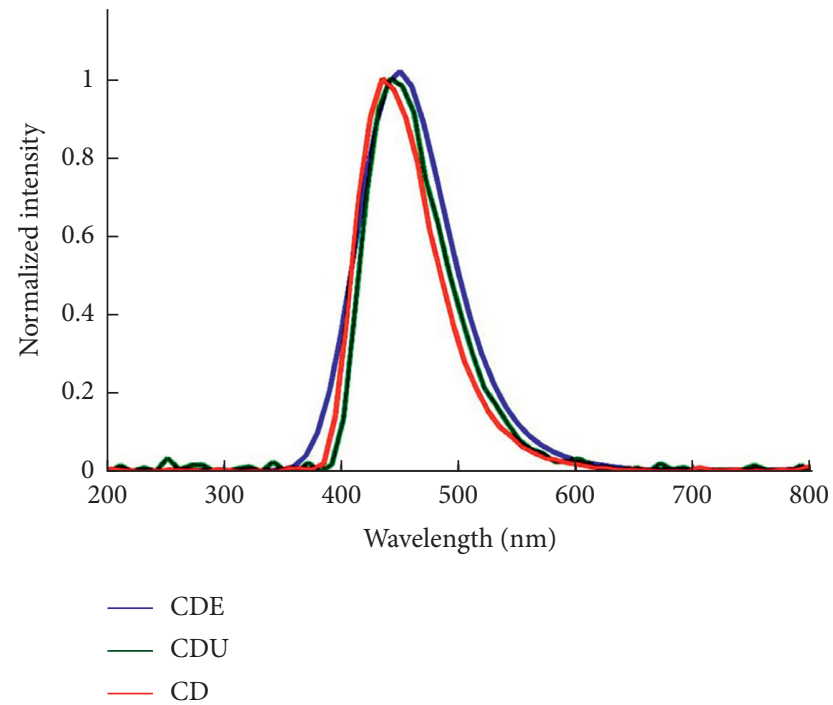

FIGURE 10: Comparison of reducing properties of CQD synthesized with urea and ethylenediamine. 


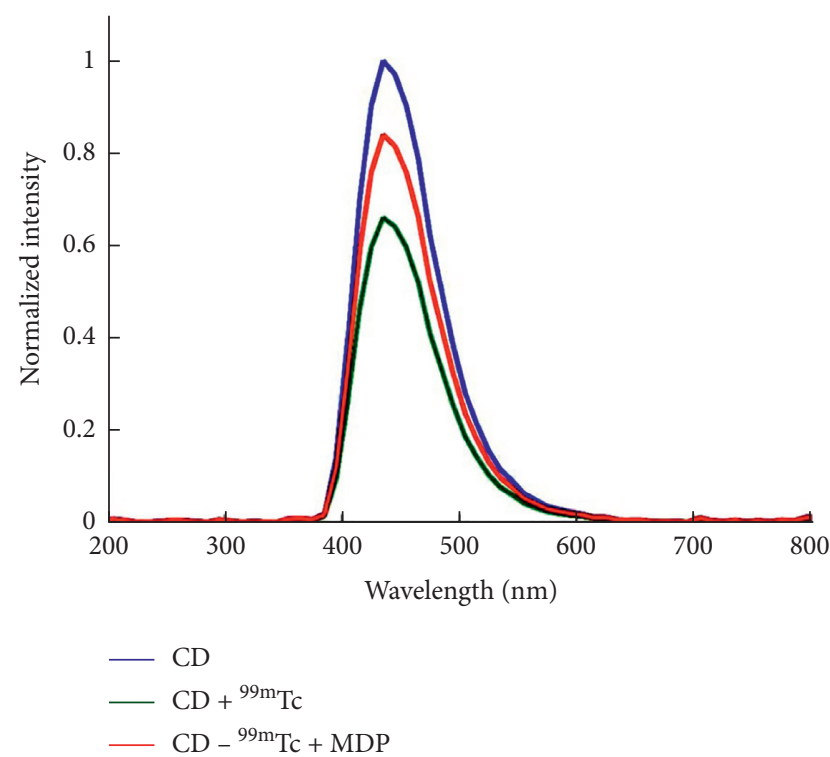

FIGURE 11: Emission spectrum of CQD synthesized by citric acid after mixing with technetium-99m and MDP.

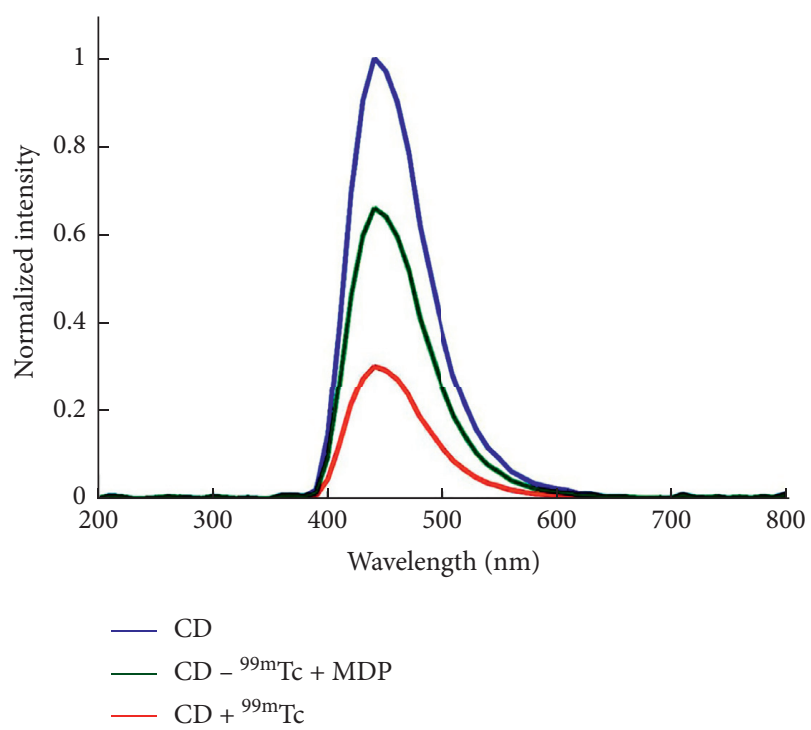

FIGURE 12: Emission spectrum of CQD synthesized by citric acid and ethylenediamine after mixing with technetium-99m and MDP.

analysis obtained from scanning electron microscopy has confirmed nitrogen, carbon, and oxygen (Figure 6). XPS analysis is shown in Figure 7, which confirmed the presence of carbon, nitrogen, and oxygen atoms. Carbon 1s region of XPS analysis showed graphitic or aliphatic carbon and also carbon-oxygen and carbon-nitrogen by appearing peaks at 283.8, $286.03 \mathrm{eV}$, and $287.55 \mathrm{eV}$, respectively (Figure 7(b))). Nitrogen 1s region showed that two peaks at $399.35 \mathrm{eV}$ and $400.29 \mathrm{eV}$ are related to aromatic nitrogen and $\mathrm{NH} 2$. Oxygen XPS analysis confirmed the presence of $\mathrm{C}=\mathrm{O}$ and $\mathrm{C}-\mathrm{O}$ in 530 and 533 , respectively.
TABLE 1: Different parameters that affect radiolabeling.

\begin{tabular}{llcccc}
\hline Factor & Name & Units & -1 & 0 & 1 \\
\hline A & MDP & mg & 5 & 10 & 15 \\
B & CDQ & ng & 5 & 10 & 15 \\
C & Time & min & 10 & 20 & 30 \\
\hline
\end{tabular}

TABle 2: Data that shows radiolabeling design.

\begin{tabular}{lcccc}
\hline Number & $\mathrm{A}$ & $\mathrm{B}$ & $\mathrm{C}$ & Percentage (\%) \\
\hline 1 & 0 & 0 & 0 & 88 \\
2 & 1 & 0 & 1 & 96 \\
3 & -1 & -1 & 0 & 82 \\
4 & 0 & -1 & -1 & 76 \\
5 & 1 & 0 & -1 & 74 \\
6 & 0 & -1 & 1 & 84 \\
7 & 0 & 1 & -1 & 81 \\
8 & 0 & 0 & 0 & 83 \\
9 & -1 & 0 & -1 & 79 \\
10 & 1 & -1 & 0 & 80 \\
11 & 0 & 0 & 0 & 85 \\
12 & 1 & 1 & 0 & 87 \\
13 & 0 & 1 & 1 & 98 \\
14 & -1 & 1 & 0 & 84 \\
15 & -1 & 0 & 1 & 91 \\
\hline
\end{tabular}

TABle 3: Variance analysis data.

\begin{tabular}{lcccc}
\hline Term & Coef & SE coef & $T$ value & $P$ value \\
\hline Constant & 77 & 1.11 & 69.33 & 0 \\
$A$ & 0 & 0.68 & 0 & 1 \\
$B$ & 3.5 & 0.68 & 5.15 & 0.004 \\
$C$ & 6.25 & 0.68 & 9.19 & 0 \\
$A^{*} A$ & -2.25 & 1 & -2.25 & 0.074 \\
$B^{*} B$ & 0.25 & 1 & 0.25 & 0.813 \\
$C^{*} C$ & -0.25 & 1 & -0.25 & 0.813 \\
$A^{*} B$ & 1 & 0.962 & 1.04 & 0.346 \\
$A^{*} C$ & 2.5 & 0.962 & 2.6 & 0.048 \\
$B^{*} C$ & 2.5 & 0.962 & 2.6 & 0.048 \\
\hline
\end{tabular}

3.2. Cytotoxicity Analysis. The MTT assay was done on the HEK-293 cell line to determine the cytotoxicity of CQD that is presented in Figure 8. Statistical analysis ( $P$ value $<0.05$ ) showed that no toxicity was observed up to $100 \mu \mathrm{g}$ compared to the control group. The assay was done in the triple set.

3.3. Determination of the Reducing Potential of CQD. Reducing properties of CQD were determined by fluorescence spectroscopy in the presence of technetium-99m, and methylene phosphonic acid was then compared with intact CQD (Figure 9). The decrease in emission intensity of CQD to $20 \%$ of its initial percentage showed the reducing properties of the nanoparticle. Also, turning the emission to $90 \%$ of its initial percentage represented the separation from the CQD surface and complex formation with methylene phosphonic acid. 

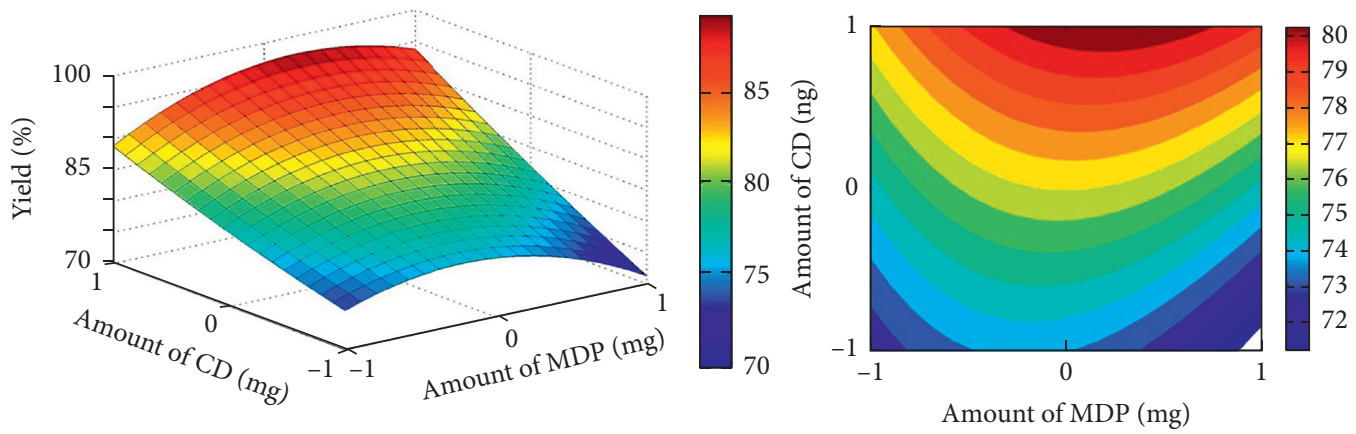

FIgURE 13: 2D and 3D images of CQD and MDP interaction.
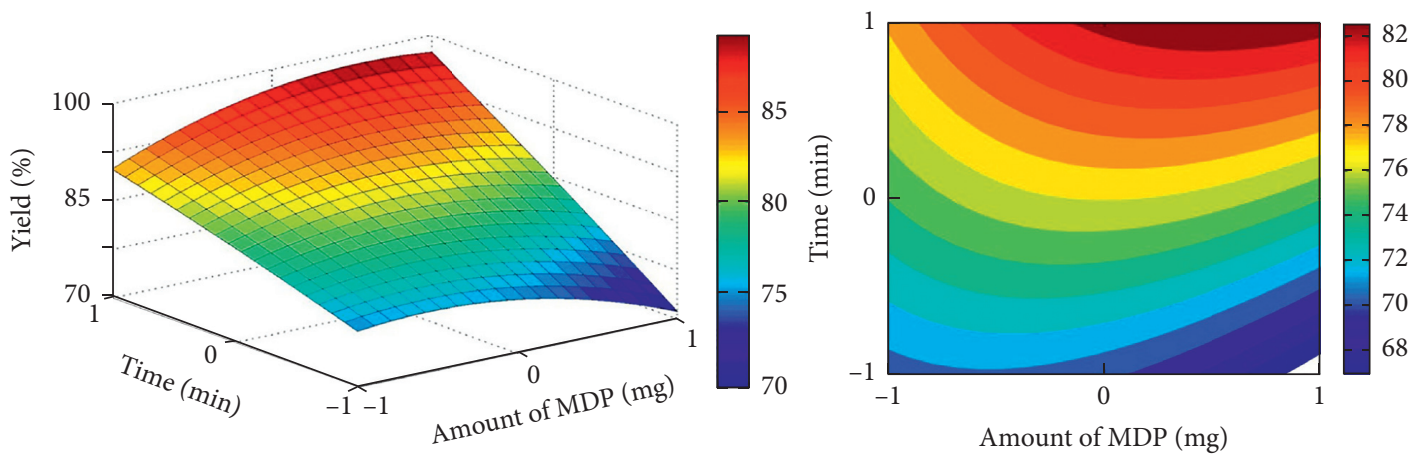

FIGURE 14: 2D and 3D images of time and MDP interaction.
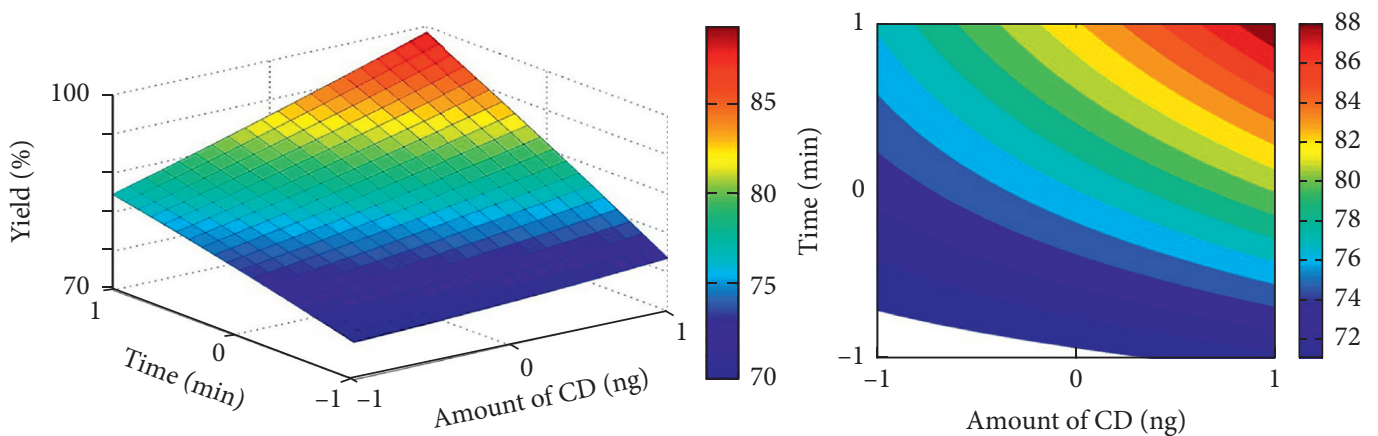

FIgURE 15: 2D and 3D images of CQD and time interaction.

3.4. Effect of Functional Group. The effect of a functional group on reducing properties in the presence of urea and ethylenediamine was evaluated. It is evident that emission at different wavelengths was changed and that the presented functional group had a useful role in electron transition. Wavelength was shifted to shorter wavelengths in the absence of a functional group while it shifted to higher wavelengths in the presence of ethylenediamine and urea. Also, ethylenediamine had more effect on wavelengths compared to urea. Furthermore, data showed that CQD synthesized with citric acid had a lower reducing effect in contrast to CQD synthesized by citric acid and ethylenediamine (Figures 10-12).

3.5. Radiolabeling. Different parameters that affect radiolabeling were considered including time, amount of ligand,
TABLE 4: Optimal amount of variables that affect radiolabeling.

\begin{tabular}{lccc}
\hline MDP $(\mathrm{mg})$ & CDQ $(\mathrm{ng})$ & Time $(\mathrm{min})$ & Max response \\
\hline 10.5 & 15 & 30 & $92(\%)$ \\
\hline
\end{tabular}

and amount of CQD (Tables 1 and 2). Statistical analysis was performed by Box-Behnken software. Variance analysis data are presented in Table 3. $P$ value $<0.05$ indicated the importance of each term. Data showed that the amount of CQD, time, ligand-time, and CQD-time interaction had a potential effect. Plus and minus represented the direct and indirect relation. 2D and 3D images show the effect of twoterm interactions in the average mean of the third term as shown in Figures 13-15. A variation in response is indicated by variation in color. Blue shows the lowest, and red shows 

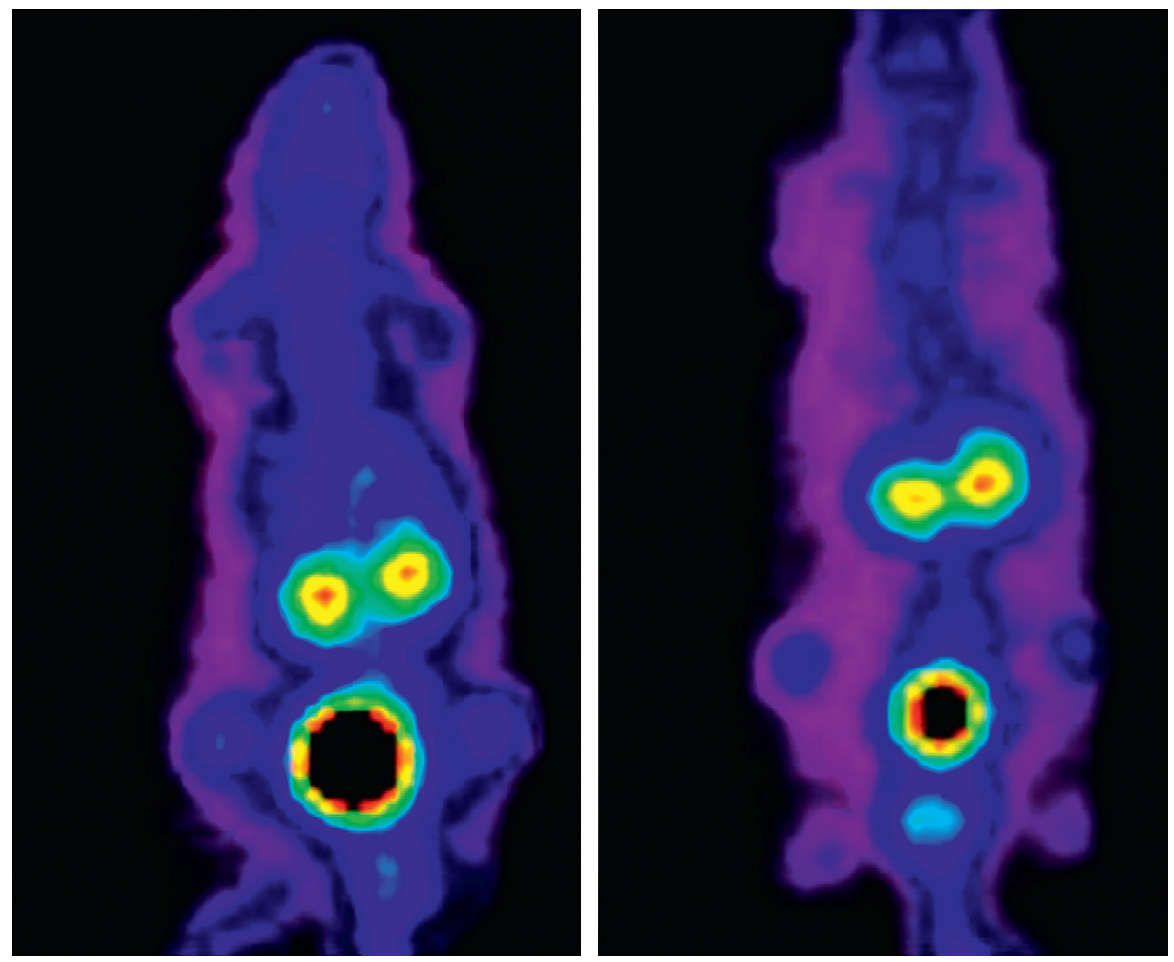

FIGURE 16: SPECT images of ${ }^{99 \mathrm{~m}}$ Tc-MDP injected in mouse in which technetium-99m was reduced with CQD.

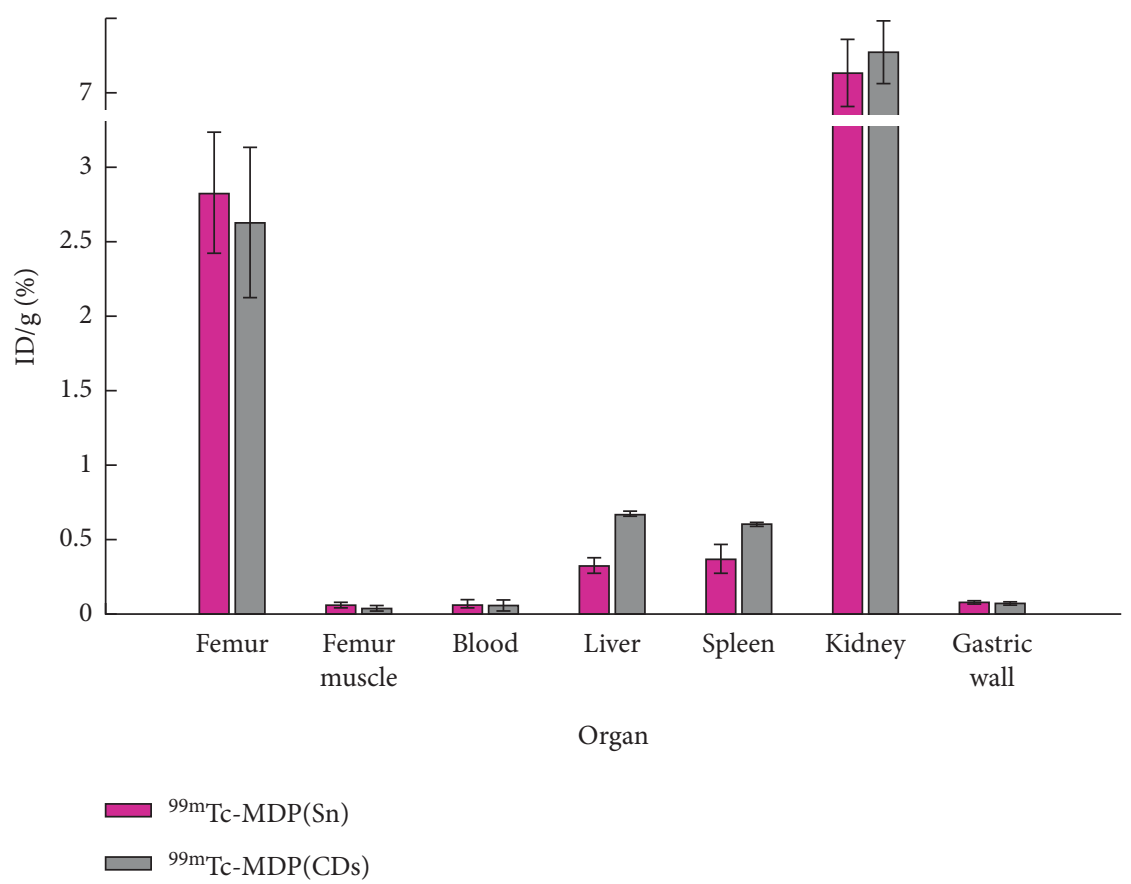

FIGURE 17: Biodistribution study of ${ }^{99 \mathrm{~m}}$ Tc-MDP injected in mouse in which technetium-99m was reduced with CQD.

the highest variation. Thus, the optimal amount of variables was calculated and is indicated in Table 4. MDP $(10.5 \mathrm{mg})$, CQD (15 ng), and time (30 min) are the optimal amount of parameters. Finally, for reducing the effect of oxygen on technetium-99m, the prepared labeling kit was lyophilized.
3.6. SPECT Imaging and Biodistribution. SPECT imaging was carried out for further study. In this regard, $3.7 \mathrm{MBq}$ of radiotracer was injected into anesthetized mice $(n=3)$. Injected mice have lied down under the SPECT instrument. The result is shown in Figure 16. A biodistribution study was performed 


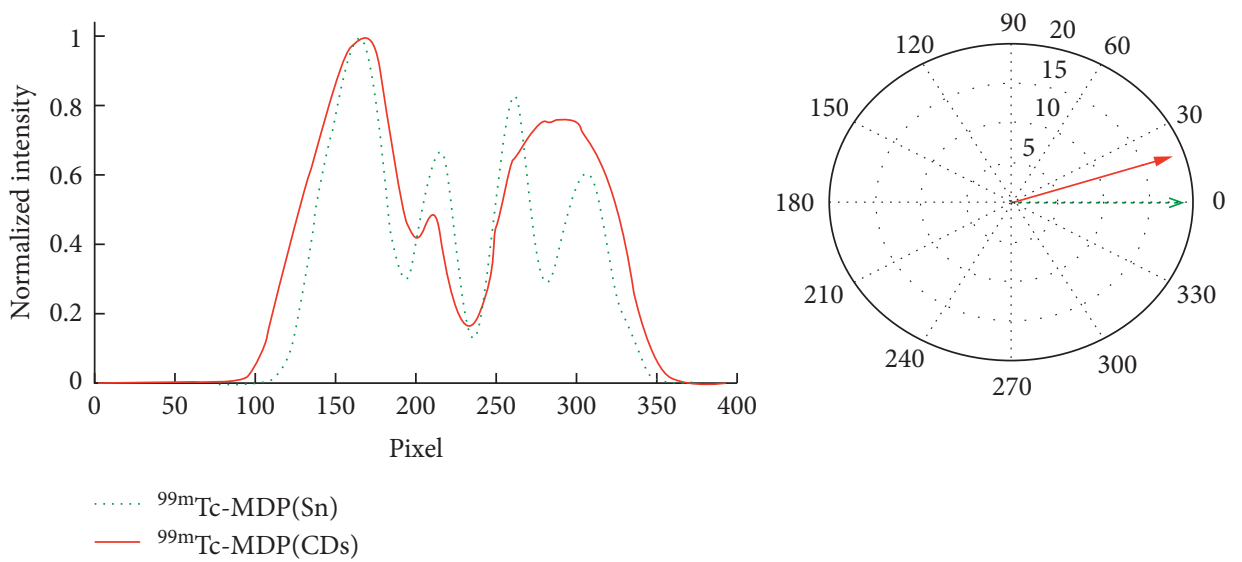

FIGURE 18: Comparison of two kits with Sn and CQD as reducing agents.

after scarifying mice. Each organ was harvested, and activity was counted with gamma well counter (Figure 17). As it is clear, high activity was observed in the femur and kidney. Accumulation of radiotracer in kidney indicted the extraction route. Acceptable radiotracer was accumulated in the femur, which indicated that technetium-99m was reduced and participated in complex formation very well. Also, radiotracer was accumulated in the liver to some extent. It means that the second extraction pathway is through the liver. For comparison of two kits (with Sn and CQD as reducing agents), images were divided into sublayers with MATLAB software, and the final vector of each layer was obtained (Figure 18). Finally, the result showed that the two images are very similar (about 90\%), and the angle between vectors is $18^{\circ}$.

The two images are approximately the same. Thus, based on the collected result, it can be deduced that two prepared kits (with Sn as reducing agent and with CQD as reducing agent) are the same, and CQD can be used as a reducing agent in the technetium-99m kit.

\section{Conclusion}

Due to the application of nanomaterials in many fields, in this study, we evaluated the reducing properties of CQD as a reducing agent in the radiopharmaceutical kit. CQD was successfully synthesized and confirmed. No cytotoxicity was observed by the MTT assay. The reducing effect of the CQD was examined and validated. All in all, fluorescent spectroscopy, SPECT imaging, and biodistribution study confirmed our hypothesis that CQD could reduce technetium-99m. Although further study must be done to determine the reducing properties of CQD on technetium-99m, our result showed that it could be used in radiopharmaceutical kit in the future.

\section{Data Availability}

The data used to support the findings of this study are available from the corresponding author upon request.

\section{Conflicts of Interest}

The authors declare that they have no conflicts of interest.

\section{References}

[1] B. Gutfilen and G. Valentini, "Radiopharmaceuticals in nuclear medicine: recent developments for SPECT and PET studies," BioMed Research International, vol. 2014, Article ID 426892, 3 pages, 2014.

[2] D. Ganguly, Medical Imaging: A Review. In Security-Enriched Urban Computing and Smart Grid, Springer, Berlin, Heidelberg, Germany, 2010.

[3] L. W. Dobrucki and A. J. Sinusas, "PET and SPECT in cardiovascular molecular imaging," Nature Reviews Cardiology, vol. 7, no. 1, pp. 38-47, 2010.

[4] K. Chen and X. Chen, "Design and development of molecular imaging probes," Current Topics in Medicinal Chemistry, vol. 10, no. 12, pp. 1227-1236, 2010.

[5] A. Boschi, L. Uccelli, and P. Martini, "A picture of modern Tc-99m radiopharmaceuticals: production, chemistry, and applications in molecular imaging," Applied Sciences, vol. 9, no. 12, p. 2526, 2019.

[6] W. F. Jiemy, P. Heeringa, J. A. A. M. Kamps, C. J. van der Laken, R. H. J. A. Slart, and E. Brouwer, "Positron emission tomography (PET) and single photon emission computed tomography (SPECT) imaging of macrophages in large vessel vasculitis: current status and future prospects," Autoimmunity Reviews, vol. 17, no. 7, pp. 715-726, 2018.

[7] S. S. Jurisson and J. D. Lydon, "Potential technetium small molecule radiopharmaceuticals," Chemical Reviews, vol. 99, no. 9, pp. 2205-2218, 1999.

[8] M. K. Dewanjee, "The chemistry of $99 \mathrm{mTc}$-labeled radiopharmaceuticals," Seminars in Nuclear Medicine, vol. 20, no. 1, pp. 5-27, 1990.

[9] F. Habashi, "Technetium, physical and chemical properties," in Encyclopedia of Metalloproteins, R. H. Kretsinger, V. N. Uversky, and E. A. Permyakov, Eds., pp. 2153-2154, Springer New York, New York, NY, USA, 2013.

[10] S. Mortazavi-Derazkola, A. Mohammad, and A. Omid, "Facile green synthesis and characterization of Crataegus microphylla extract-capped silver nanoparticles (CME@Ag-NPs) and its potential antibacterial and anticancer activities against AGS and MCF-7 human cancer cells," Journal of Alloys and Compounds, vol. 820, Article ID 153186, 2020.

[11] M. Ebrahimzadeh, S. Mortazavi, and M. Zazouli, "Ecofriendly green synthesis and characterization of novel Fe3O4/ $\mathrm{SiO} 2 / \mathrm{Cu} 2 \mathrm{O}-\mathrm{Ag}$ nanocomposites using Crataegus pentagyna fruit extract for photocatalytic degradation of organic 
contaminants," Journal of Materials Science: Materials in Electronics, vol. 30, no. 12, 2019.

[12] H. Khojasteh, H. Safajou, S. Mortazavi-Derazkola, M. Salavati-Niasari, K. Heydaryan, and M. Yazdani, "Economic procedure for facile and eco-friendly reduction of graphene oxide by plant extracts; a comparison and property investigation," Journal of Cleaner Production, vol. 229, pp. 1139-1147, 2019.

[13] M. A. Ebrahimzadeh, A. Naghizadeh, O. Amiri, M. ShirzadiAhodashti, and S. Mortazavi-Derazkola, "Green and facile synthesis of Ag nanoparticles using Crataegus pentagyna fruit extract (CP-AgNPs) for organic pollution dyes degradation and antibacterial application," Bioorganic Chemistry, vol. 94, Article ID 103425, 2020.

[14] J. K. Patra, D. Gitishree, F. F. Leonardo, V. R. C. Estefania et al., "Nano based drug delivery systems: recent developments and future prospects," Journal of Nanobiotechnology, vol. 16, no. 1, p. 71, 2018.

[15] W. H. De Jong and P. J. A. Borm, "Drug delivery and nanoparticles: applications and hazards," International Journal of Nanomedicine, vol. 3, no. 2, pp. 133-149, 2008.

[16] A. Z. Wilczewska, K. Niemirowicz, K. H. Markiewicz, and H. Car, "Nanoparticles as drug delivery systems," Pharmacological Reports, vol. 64, no. 5, pp. 1020-1037, 2012.

[17] O. Salata, "Applications of nanoparticles in biology and medicine," Journal of Nanobiotechnology, vol. 2, no. 1, p. 3, 2004.

[18] P. Padmanabhan, A. Kumar, S. Kumar, R. K. Chaudhary, and B. Gulyás, "Nanoparticles in practice for molecular-imaging applications: an overview," Acta Biomaterialia, vol. 41, pp. 1-16, 2016.

[19] M. Gholinejad, S. Mohammad, R. Mehran, N. Carmen, and K.-Z. Mohsen, "Iron oxide nanoparticles modified with carbon quantum nanodots for the stabilization of palladium nanoparticles: an efficient catalyst for the suzuki reaction in aqueous media under mild conditions," ChemCatChem, vol. 8, no. 2, 2015.

[20] M. Amjadi, T. Hallaj, H. Asadollahi, Z. Song, M. de Frutos, and N. Hildebrandt, "Facile synthesis of carbon quantum dot/ silver nanocomposite and its application for colorimetric detection of methimazole," Sensors and Actuators B: Chemical, vol. 244, pp. 425-432, 2017.

[21] T. Liu, J. X. Dong, S. G. Liu et al., "Carbon quantum dots prepared with polyethyleneimine as both reducing agent and stabilizer for synthesis of Ag/CQDs composite for $\mathrm{Hg}^{2+}$ ions detection," Journal of Hazardous Materials, vol. 322, pp. 430-436, 2017.

[22] J. Song, L. Zhao, Y. Wang et al., "Carbon quantum dots prepared with chitosan for synthesis of CQDs/AuNPs for iodine ions detection," Nanomaterials, vol. 8, no. 12, p. 1043, 2018.

[23] Y. Rai, P. Richa, K. Neeraj, K. S. Dhananjay et al., "Mitochondrial biogenesis and metabolic hyperactivation limits the application of MTT assay in the estimation of radiation induced growth inhibition," Scientific Reports, vol. 8, no. 1, p. 1531, 2018.

[24] J. van Meerloo, G. J. L. Kaspers, and J. Cloos, "Cell sensitivity assays: the MTT assay," in Cancer Cell Culture: Methods and Protocols, I., A. Cree, Ed., pp. 237-245, Humana Press, Totowa, NJ, USA, 2011.

[25] A. Chae, Y. Choi, S. Jo et al., "Microwave-assisted synthesis of fluorescent carbon quantum dots from an A2/B3 monomer set," RSC Advances, vol. 7, no. 21, pp. 12663-12669, 2017. 